\title{
Calibrating Wrist-Worn Accelerometers for Physical Activity Assessment in Preschoolers: Machine Learning Approaches
}

Shiyu Li ${ }^{1}$, MSc; Jeffrey T Howard ${ }^{2}, \mathrm{PhD}$; Erica T Sosa ${ }^{2}, \mathrm{PhD}$; Alberto Cordova ${ }^{3}, \mathrm{PhD}$; Deborah Parra-Medina ${ }^{4}, \mathrm{PhD}$; Zenong $\mathrm{Yin}^{2}, \mathrm{PhD}$

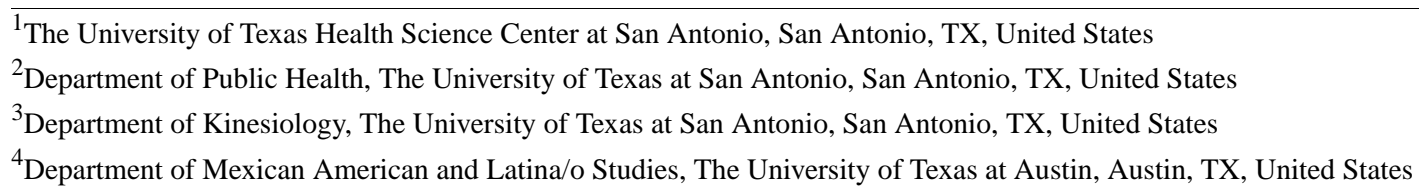

Corresponding Author:

Shiyu Li, MSc

The University of Texas Health Science Center at San Antonio

7703 Floyd Curl Dr

San Antonio, TX, 78229

United States

Phone: 12102047621

Email: lis9@ livemail.uthscsa.edu

\section{Abstract}

Background: Physical activity (PA) level is associated with multiple health benefits during early childhood. However, inconsistency in the methods for quantification of PA levels among preschoolers remains a problem.

Objective: This study aimed to develop PA intensity cut points for wrist-worn accelerometers by using machine learning (ML) approaches to assess PA in preschoolers.

Methods: Wrist- and hip-derived acceleration data were collected simultaneously from 34 preschoolers on 3 consecutive preschool days. Two supervised ML models, receiver operating characteristic curve (ROC) and ordinal logistic regression (OLR), and one unsupervised ML model, k-means cluster analysis, were applied to establish wrist-worn accelerometer vector magnitude (VM) cut points to classify accelerometer counts into sedentary behavior, light PA (LPA), moderate PA (MPA), and vigorous PA (VPA). Physical activity intensity levels identified by hip-worn accelerometer VM cut points were used as reference to train the supervised ML models. Vector magnitude counts were classified by intensity based on three newly established wrist methods and the hip reference to examine classification accuracy. Daily estimates of PA were compared to the hip-reference criterion.

Results: In total, 3600 epochs with matched hip- and wrist-worn accelerometer VM counts were analyzed. All ML approaches performed differently on developing PA intensity cut points for wrist-worn accelerometers. Among the three ML models, k-means cluster analysis derived the following cut points: $\leq 2556$ counts per minute $(\mathrm{cpm})$ for sedentary behavior, 2557-7064 cpm for LPA, 7065-14532 cpm for MPA, and $\geq 14533 \mathrm{cpm}$ for VPA; in addition, k-means cluster analysis had the highest classification accuracy, with more than $70 \%$ of the total epochs being classified into the correct PA categories, as examined by the hip reference. Additionally, k-means cut points exhibited the most accurate estimates on sedentary behavior, LPA, and VPA as the hip reference. None of the three wrist methods were able to accurately assess MPA.

Conclusions: This study demonstrates the potential of ML approaches in establishing cut points for wrist-worn accelerometers to assess PA in preschoolers. However, the findings from this study warrant additional validation studies.

(JMIR Form Res 2020;4(8):e16727) doi: 10.2196/16727

\section{KEYWORDS}

preschoolers; accelerometer; physical activity; obesity; machine learning 


\section{Introduction}

Accelerometry has been widely accepted as the gold standard to measure physical activity (PA) in free-living settings [1,2] including preschools [3]. Triaxial accelerometers can record the magnitude of accelerations from three movement axes and convert accelerations to vector magnitude counts over a given user-specified cycling period (epoch) [4]. Counts are translated into biologically meaningful PA volume and intensity levels using pre-established cut points for sedentary behavior, light physical activity (LPA), moderate physical activity (MPA), and vigorous physical activity (VPA) [3]. Although; traditionally, gold standard cut points are established using data derived from accelerometers placed on the right hip of the body [1,2], recent revolutions have focused on acceleration data from wrist-worn accelerometers for increased protocol compliance of study participants, better sensitivity to detect certain types of movements, and sleep measurement [5-9]. Fairclough et al [10] found that wrist-worn accelerometers had at least $10 \%$ higher compliance rate than hip-worn ones, regardless of the data processing criteria in school-age children. They also reported that wrist-worn accelerometers had a much lower study drop-off rate compared to hip-worn ones, regardless of the number of monitoring days. Thus, the wrist, instead of the hip, might be an ideal accelerometer placement site for preschoolers.

However, cut points from wrist-derived data are sparse for preschool-age children [11,12]. Johansson and colleagues $[13,14]$ conducted the only studies that established wrist-referenced cut points for sedentary behavior and moderate and vigorous physical activity (MVPA) in preschoolers. Using direct observation of structured and free-play activities as the ground truth activities, hip- and wrist-derived cut points yielded comparable accuracy and validity of the observed activities. Nevertheless, Johansson [15] cut points did not differentiate between LPA, MPA, and VPA, and have not been replicated by others.

Machine learning (ML) algorithms are increasingly being used to translate accelerometer outputs to meaningful PA metrics [16]. Recent studies have applied part of the accelerometry data to $\mathrm{ML}$ algorithms as the training set to build statistical models that can predict PA intensities from a new set of accelerometry data without explicit instructions [17]. Research has demonstrated the promising performance of ML techniques in combination with the use of wrist-derived acceleration data in predicting the type and intensity of activities, as well as activity energy expenditure with comparable overall predictive accuracies in adult populations $[16,18,19]$.

Combining ML techniques and wrist-worn accelerometers may help address the low compliance caused by the challenges in wearing hip-worn accelerometers [20,21] and the difficulty in measuring the various levels of activity intensity given the unique nature of the sporadic and short-burst activity patterns in preschool-age children [22,23]. Therefore, the purposes of this study were (1) to develop the cut point values for sedentary behavior, LPA, MPA, and VPA based on wrist-derived acceleration data using multiple ML algorithms, and (2) to examine classification accuracy of PA intensity in comparison to hip-reference cut points [24] and previously established wrist-referenced cut points [25] in preschool-age children.

\section{Methods}

\section{Recruitment}

A total of 61 healthy children, aged 3-5 years, who were enrolled in a Head Start program (HS), were recruited to participate in the study in Fall 2018; in San Antonio, Texas. The HS is a federally funded program that serves children from low-income families through academic, health, nutrition, and family service programs [26]. Their parents/guardians signed an informed consent form before participation. Each participant received up to US $\$ 30$ gift card for participating in the study. Children who were 3 years old at recruitment, were enrolled in the full-day HS program, and had no physical disabilities were eligible for the study.

This study was reviewed and approved by the Institutional Review Board of the University of Texas at San Antonio.

\section{Accelerometer Data Collection}

For 3 consecutive days, the children wore two triaxial accelerometers (ActiGraph model WGT3X-BT, ActiGraph, LLC) that collected raw accelerometry data at a sampling frequency of $30 \mathrm{~Hz}$ ( 30 observations per second for each axis) from 9 AM to $2 \mathrm{PM}$, following a previously published protocol [27]. On the day of data collection, a trained research assistant placed one accelerometer on the nondominant wrist and the other one on the right hip of each child. For this study, raw accelerations were converted into 15-second epoch and thereafter collapsed to 60-second epochs. For this study, data were outputted as the vector magnitude (VM) counts, which is the square root of the sum of squares of each axis of acceleration data. Nonwear time was detected using the Choi wear time validation algorithm [28]. Participants with missing hip- or wrist-worn accelerometer epoch for more than 3 consecutive 5-hour days were excluded from the analysis. Accelerometer data processing was performed using ActiLife software (Version 6.13.3). Visual presentation of the accelerometer counts for one participant is shown in Figure 1. 
Figure 1. Visual presentation of the changes of wrist- and hip-worn accelerometer vector magnitude counts for one participant throughout a school day, with previously established hip-based physical activity level thresholds as defined by Butte et al. [23].

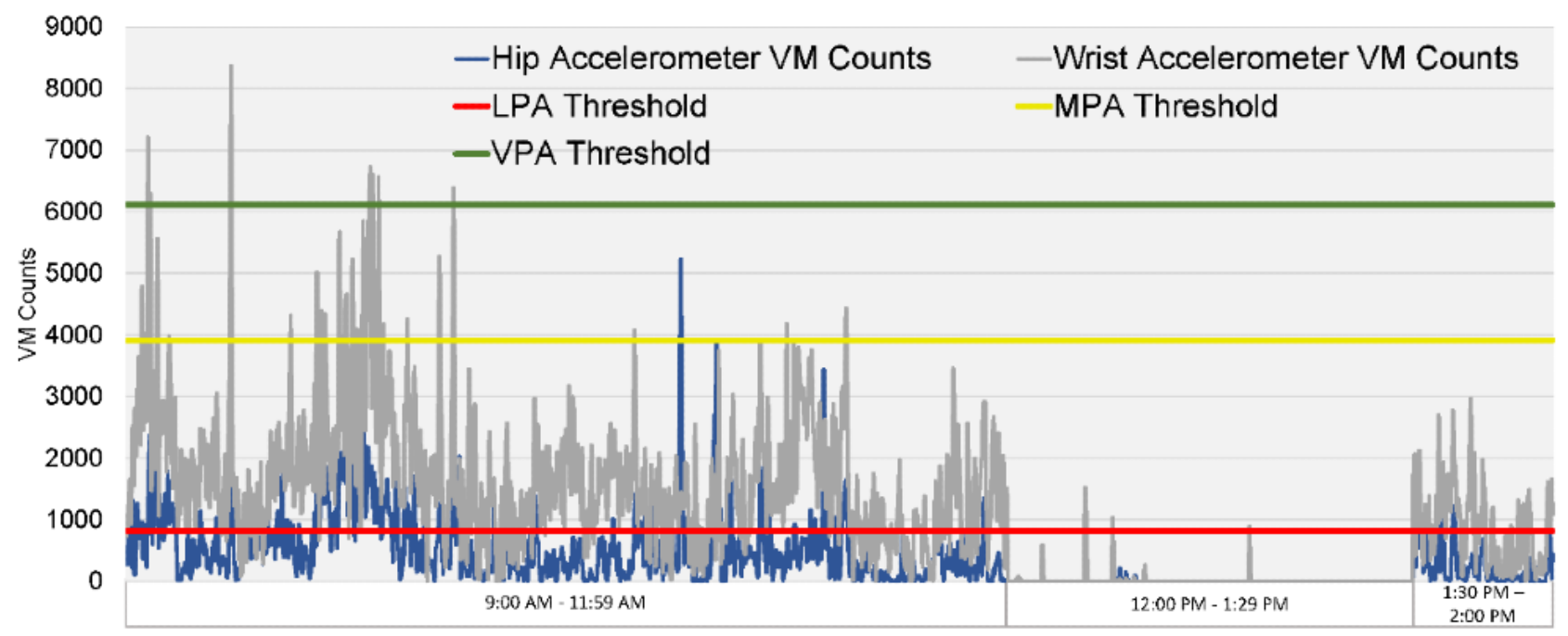

Time Over One School Day

\section{Hip- and Wrist-Reference Cut Points for Comparisons}

The hip-reference cut points for assessing PA intensity were adopted from Butte et al [24] based on predicted energy expenditure from room calorimetry and doubly labeled water. The cut points were no more than 820 counts per minute (cpm) for sedentary behavior, 821-3908 cpm for LPA, 3909-6112 cpm for MPA, and greater than $6113 \mathrm{cpm}$ for VPA for vector magnitude counts collected from ActiGraph hip-worn accelerometers during free-living activities in preschool-age children. The cut points reported by Butte and colleagues [13] are widely used and will be used as the gold-standard reference in this study. The cut points for sedentary behavior and MVPA from wrist-derived data captured during structured and free-play activities by Johansson et al [14] are the only available references for preschool-age children.

\section{Applications of Machine Learning Techniques}

Three ML models, two supervised and one unsupervised, were used to establish three different sets of wrist-worn accelerometer VM cut points as the new wrist methods to assess PA in preschoolers (Figure 2). Supervised ML models learn from hip-identified PA of each epoch and produce an inferred function that maps the wrist accelerometer count to a PA category; unsupervised models read the underlying structure of the wrist-worn accelerometer counts and detect the PA level of each count value [29]. PA intensity levels identified using hip-worn accelerometer cut points by Butte et al [24] were used as the hip reference to train the supervised ML models. The two supervised ML techniques were receiver operating characteristic (ROC) analysis and the ordinal logistic regression (OLR) model. Since ROC analysis was designed to predict binary outcomes, it was run three times to establish the upper threshold for sedentary behavior and the lower thresholds for LPA and VPA [30]. After ROC analysis calculated and compared sensitivity values for all possible threshold values, we selected thresholds based on the minimum difference between sensitivity and specificity [31]. For the OLR method, after being trained by the PA intensity levels as predicted by the hip reference, the newly constructed model calculated and compared the probability of each VM count value being classified into different PA intensity levels and assigned each count to the PA level with the highest predicted probability. K-means cluster analysis was the unsupervised learning approach used to separate each 15-second epoch for each participant into four distinct clusters, based solely on the input VM count data [32-34]. The number of clusters $(\mathrm{k}=4)$ was determined a priori because the four activity states of sedentary behavior, LPA, MPA, and VPA were known. Sedentary behavior cut points for OLR and k-means cluster analysis were determined based on the maximum count value within the sedentary behavior category; MPA and VPA cut points were determined based on the minimum count values within these two PA intensity categories; LPA was further determined based on the sedentary behavior and MPA cut points. 
Figure 2. Modeling process diagram.

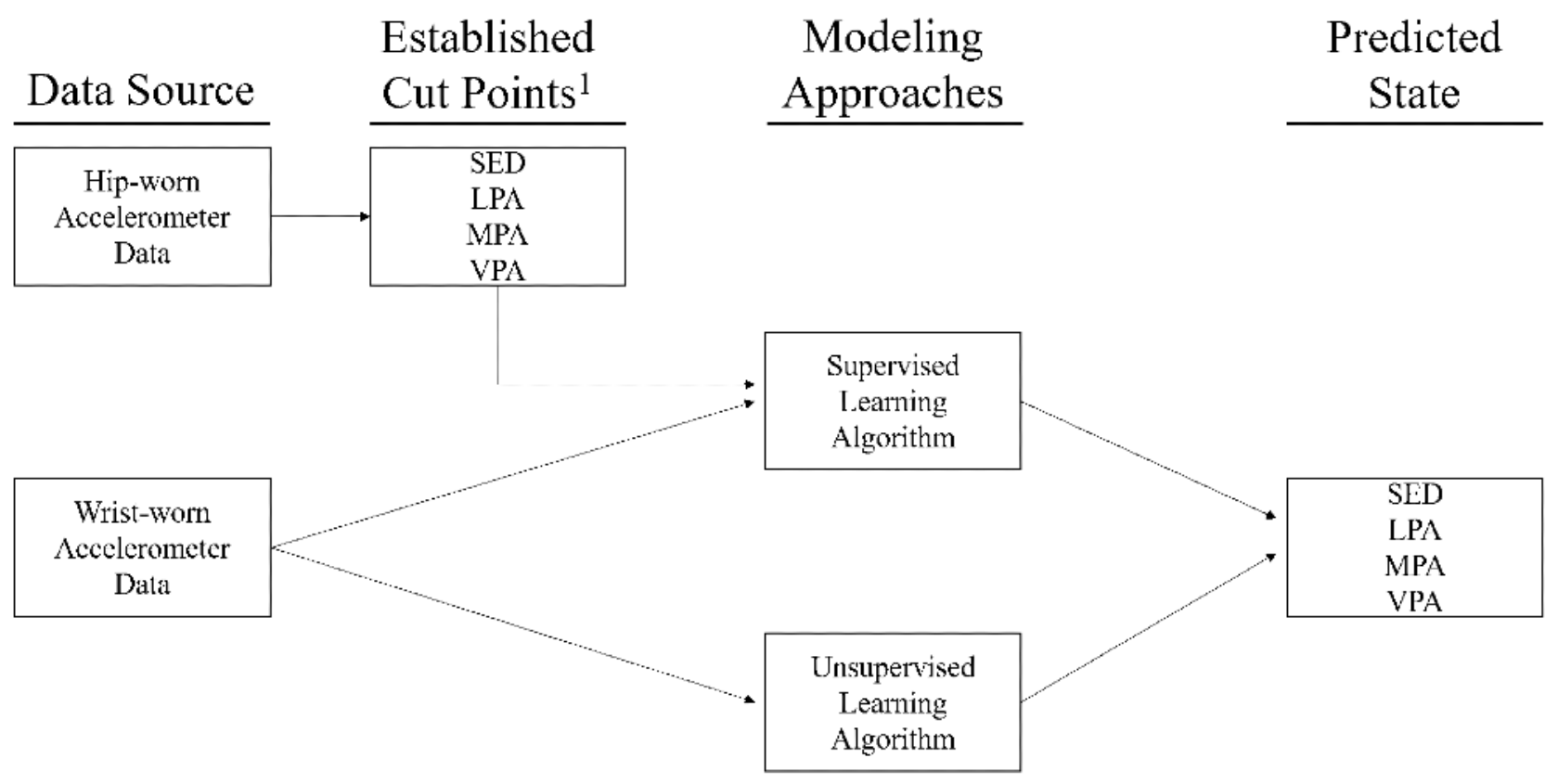

${ }^{1}$ Butte et al. (2013) VM Cut Points

\section{Statistical Analysis}

The VM count for each epoch for each participant was categorized into a PA level based on each of the three sets of newly established wrist-worn accelerometer cut points and the hip reference, resulting in four separate PA level designations. Standard classification measures of sensitivity, specificity, false-negative rate, and false-positive rate were calculated to assess the discriminative ability of each method to correctly classify PA levels. Cohen kappa values were calculated to test the agreement between hip- and four wrist-derived measures, k-means, ROC, OLR, and Johansson's cut points. Daily amount of time in each PA level was also calculated and compared against PA estimates from the hip-worn accelerometer.
Univariate analysis of covariance was conducted to compare mean daily time in each PA intensity level for each of the wrist-worn ML-based cut points versus the hip reference and the Johansson's cut points.

\section{Results}

All study participants were 3-5 years old, and more than $80 \%$ (29/34) of them were of Hispanic ethnicity (Table 1). Hip accelerometer identified $64.2 \%$ activity counts representing sedentary behavior and nearly $8.0 \%$ representing MVPA. Matched hip- and wrist-worn accelerometer data were collected and analyzed from 34 participants, yielding a total of 122,399 epochs (Figure 3 ).

Table 1. Descriptive characteristics of the study participants ( $N=34)$.

\begin{tabular}{ll}
\hline Variables & Total \\
\hline Female, $\mathrm{n}(\%)$ & $20(58.8)$ \\
Hispanic race, $\mathrm{n}(\%)$ & $29(85.3)$ \\
Age (years), mean (SD) & $3.97(0.49)$ \\
Height (cm), mean (SD) & $100.80(4.70)$ \\
Weight (kg), mean (SD) & $16.38(2.157)$ \\
Hip-based activity counts in each physical activity level, n (\%) & \\
$\quad$ Sedentary behavior & $78,538(64.2)$ \\
$\quad$ Light physical activity & $34,243(28.0)$ \\
$\quad$ Moderate physical activity & $6784(5.5)$ \\
$\quad$ Migorous physical activity & $2834(2.3)$ \\
\hline
\end{tabular}


Figure 3. Data flow diagram.

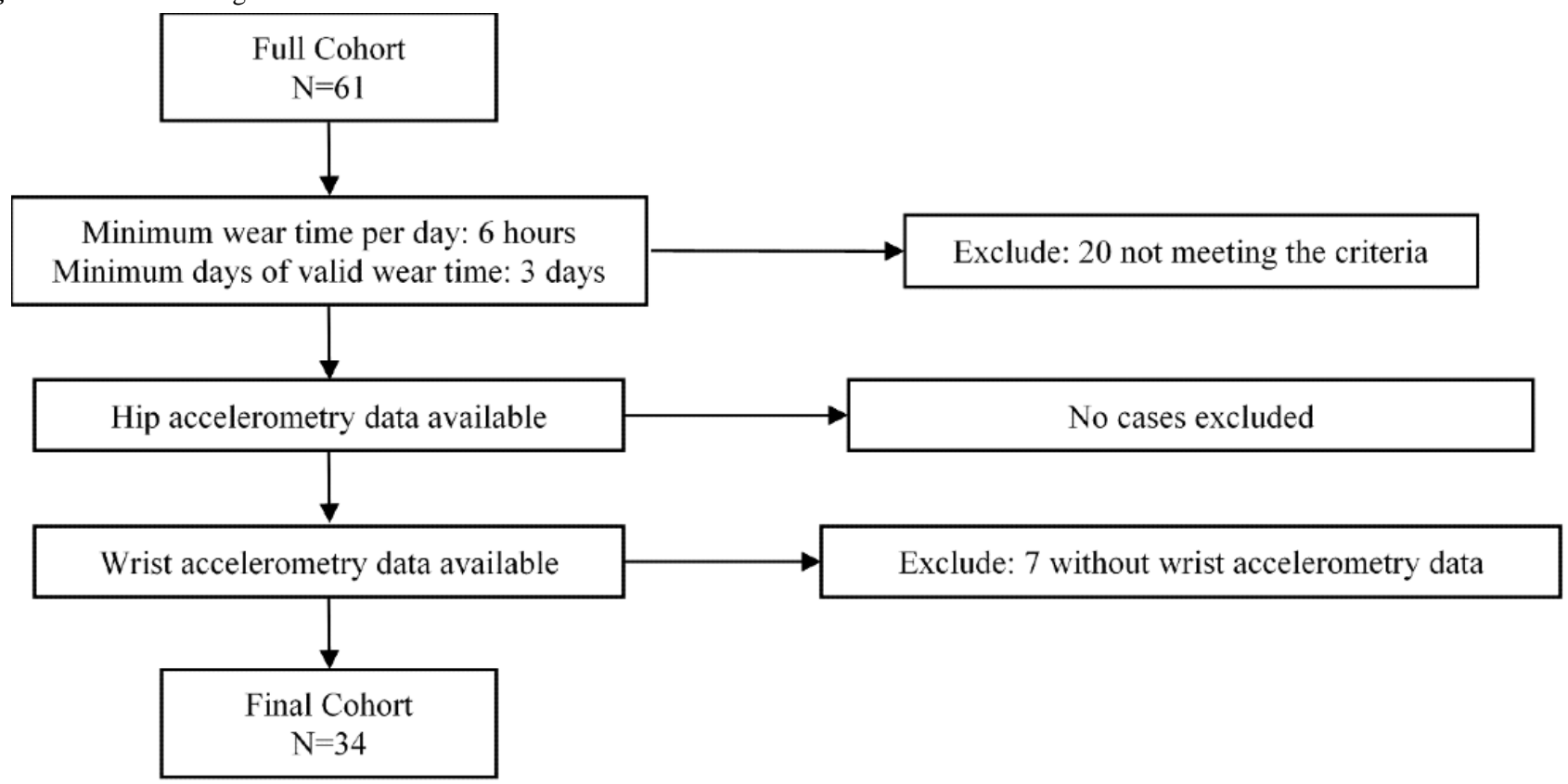

The three ML models grouped accelerometer counts differently and developed three sets of wrist-worn accelerometer VM cut mean count values increased as the PA intensity level increased, points (lower and upper bounds; Table 2). For each ML model,

Table 2. Wrist-worn accelerometer VM cut points established by each ML model and mean count value within each PA category.

\begin{tabular}{|c|c|c|c|c|}
\hline \multirow[t]{2}{*}{ Model } & \multirow[t]{2}{*}{$\mathrm{N}$} & \multicolumn{2}{|c|}{ Cut points $\left(\mathrm{cpm}^{\mathrm{a}}\right)$} & \multirow{2}{*}{$\begin{array}{l}\text { Vector magnitude counts (cpm) } \\
\text { Mean (SD) }\end{array}$} \\
\hline & & Lower Bound & Upper Bound & \\
\hline \multicolumn{5}{|c|}{ Receiver operating characteristic analysis } \\
\hline Sedentary behavior & 70,848 & 0 & 3406 & $744.40(1077.67)$ \\
\hline Light physical activity & 20,194 & 3407 & 5690 & $4509.79(652.33)$ \\
\hline Moderate physical activity & 4009 & 5691 & 6219 & $5952.98(150.75)$ \\
\hline Vigorous physical activity & 27,348 & 6220 & $\infty$ & $10,054.39(4267.24)$ \\
\hline \multicolumn{5}{|l|}{ Ordinal logistic regression model } \\
\hline Sedentary behavior & 92,143 & 0 & 5837 & $1629.59(1900.25)$ \\
\hline Light physical activity & 26,746 & 5838 & 14,020 & $8436.16(2028.01)$ \\
\hline Moderate physical activity & 1688 & 14,021 & 17,432 & $15,481.18(968.54)$ \\
\hline Vigorous physical activity & 1822 & 17,433 & $\infty$ & $22,350.31(4593.46)$ \\
\hline \multicolumn{5}{|l|}{ K-means analysis } \\
\hline Sedentary behavior & 62,815 & 0 & 2556 & 457.57 (759.38) \\
\hline Light physical activity & 37,876 & 2557 & 7067 & $4655.99(1270.28)$ \\
\hline Moderate physical activity & 18,559 & 7068 & 14,535 & 9474.95 (1895.16) \\
\hline Vigorous physical activity & 3149 & 14,536 & $\infty$ & $19,595.06(4787.10)$ \\
\hline
\end{tabular}

${ }^{\mathrm{a}} \mathrm{cpm}$ : counts per minute.

\section{Agreement Between Each Wrist Method and the Hip Reference}

Agreement between the wrist methods and the hip reference at epoch level is presented at Table 3. When grouping the MPA and VPA, the prediction accuracy of each wrist method was more comparable with the hip reference, based on a higher classification rate and kappa value. 
Table 3. Agreement of each wrist cut point compared to the hip reference.

\begin{tabular}{|c|c|c|c|c|}
\hline Activity intensity & Johansson Wrist & $\mathrm{ROC}^{\mathrm{a}}$ analysis & OLR $^{\mathrm{b}}$ model & $\mathrm{K}$-means analysis \\
\hline \multicolumn{5}{|l|}{ Sedentary behavior } \\
\hline Sensitivity (\%) & 81.36 & 77.82 & 90.95 & 71.64 \\
\hline Specificity $(\%)$ & 72.77 & 77.82 & 52.78 & 85.07 \\
\hline FPR $(\%)$ & 27.23 & 22.18 & 47.22 & 14.93 \\
\hline $\operatorname{FNR}(\%)$ & 18.64 & 22.18 & 9.05 & 28.36 \\
\hline Kappa & 0.53 & 0.54 & 0.47 & 0.53 \\
\hline \multicolumn{5}{|l|}{ Light physical activity } \\
\hline Sensitivity (\%) & 67.34 & 26.80 & 43.27 & 50.90 \\
\hline Specificity (\%) & 75.69 & 87.50 & 86.47 & 76.81 \\
\hline FPR $(\%)$ & 24.31 & 12.50 & 56.73 & 23.19 \\
\hline $\operatorname{FNR}(\%)$ & 32.66 & 73.20 & 13.53 & 49.10 \\
\hline Kappa & 0.39 & 0.16 & 0.32 & 0.27 \\
\hline \multicolumn{5}{|l|}{ Moderate physical activity } \\
\hline Sensitivity (\%) & $N / A^{c}$ & 4.47 & 8.83 & 48.42 \\
\hline Specificity $(\%)$ & N/A & 96.79 & 99.06 & 86.79 \\
\hline $\operatorname{FPR}(\%)$ & N/A & 3.21 & 0.96 & 13.21 \\
\hline FNR $(\%)$ & N/A & 95.53 & 76.11 & 51.58 \\
\hline Kappa & N/A & 0.02 & 0.12 & 0.19 \\
\hline \multicolumn{5}{|l|}{ Vigorous physical activity } \\
\hline Sensitivity $(\%)$ & N/A & 79.00 & 23.89 & 33.94 \\
\hline Specificity $(\%)$ & N/A & 79.00 & 99.04 & 98.17 \\
\hline $\operatorname{FPR}(\%)$ & N/A & 21.00 & 0.96 & 1.83 \\
\hline FNR $(\%)$ & N/A & 21.00 & 76.11 & 66.06 \\
\hline Kappa & N/A & 0.11 & 0.28 & 0.31 \\
\hline \multicolumn{5}{|c|}{ Moderate-to-vigorous physical activity } \\
\hline Sensitivity $(\%)$ & 16.02 & 78.89 & 24.27 & 68.37 \\
\hline Specificity $(\%)$ & 99.53 & 78.92 & 98.96 & 86.58 \\
\hline $\operatorname{FPR}(\%)$ & 0.47 & 21.08 & 1.04 & 13.42 \\
\hline FNR $(\%)$ & 83.98 & 21.11 & 75.73 & 31.63 \\
\hline Kappa & 0.24 & 0.28 & 0.33 & 0.35 \\
\hline \multicolumn{5}{|l|}{ Overall agreement } \\
\hline Correct classification (\%) & N/A & 59.51 & 71.51 & 63.68 \\
\hline Kappa & N/A & 0.30 & 0.37 & 0.37 \\
\hline \multicolumn{5}{|c|}{ Overall agreement when MPA and VPA were grouped together } \\
\hline Correct classification (\%) & 72.30 & 63.63 & 72.37 & 65.58 \\
\hline Kappa & 0.45 & 0.35 & 0.39 & 0.40 \\
\hline
\end{tabular}

${ }^{\mathrm{a}} \mathrm{ROC}$ : Receiver operating characteristic.

${ }^{\mathrm{b}}$ OLR: Ordinal logistic regression.

${ }^{\mathrm{c}}$ N/A: Not applicable.

According to the kappa values, all three ML models performed well on identifying sedentary behavior. When compared against the hip reference, ROC analysis and k-means cluster analysis derived cut points with acceptable sensitivity and specificity values (both $>70 \%$ ) and were comparable to the performance of Johansson et al [25] cut points. 
In terms of classifying LPA, only the k-means LPA cut point resulted with sensitivity and specificity values greater than $50 \%$. While the specificity value for k-means LPA cut point $(76.81 \%)$ was similar to the Johansson cut point (75.69\%), it had a much lower sensitivity value.

None of the three wrist methods were able to distinguish MPA as indicated by the low sensitivity and kappa values, although the k-means MPA cut point had the highest sensitivity $(48.42 \%)$ and kappa value (0.19). OLR and k-means cut points also had low sensitivity values for identifying VPA (23.89\% for OLR, 33.94 for k-means). Although the ROC cut point exhibited sensitivity and specificity values of $79 \%$, the low kappa value (0.11) indicated that there was a low agreement between this method and the hip reference. When MPA and VPA were grouped together, the k-means cut points demonstrated higher sensitivity $(68.37 \%)$, specificity $(86.58 \%)$, and kappa values $(0.35)$ than the other two wrist methods.
In general, k-means cut points resulted in sensitivity and specificity values above $50 \%$ for predicting sedentary behavior, LPA, and MVPA, with an acceptable kappa value for overall agreement (0.40).

\section{Physical Activity Estimates by Method}

Table 4 presents daily amount of time and percent of time in each PA intensity level as assessed by the hip reference, Johansson et al [25] wrist VM cut points, and the three newly developed wrist methods. ROC and k-means sedentary behavior cut points were close to the hip reference on estimating sedentary behavior time. LPA estimates were similar among the Johansson et al [25] cut point, the hip reference, and the k-means wrist method. None of the three wrist methods were comparable to the hip reference on estimating MPA and MVPA. Univariate ANOVA showed a similar VPA estimates for the $\mathrm{k}$-means wrist method and the hip reference.

Table 4. Daily time in each Physical Activity intensity level (\%) by different sets of cut points.

\begin{tabular}{|c|c|c|c|c|c|c|c|c|c|c|}
\hline \multirow[t]{2}{*}{ Activity intensity } & \multicolumn{2}{|c|}{ Hip reference } & \multicolumn{2}{|c|}{ Johansson wrist } & \multicolumn{2}{|c|}{ ROC $^{\mathrm{a}}$ analysis } & \multicolumn{2}{|l|}{ OLR $^{b}$ model } & \multicolumn{2}{|c|}{$\mathrm{K}$-means analysis } \\
\hline & Mean (SD) & $\%$ & Mean (SD) & $\%$ & Mean (SD) & $\%$ & Mean (SD) & $\%$ & Mean (SD) & $\%$ \\
\hline Sedentary behavior & $\begin{array}{l}148.07 \\
(32.15)\end{array}$ & $57.8^{\mathrm{c}}$ & $\begin{array}{l}194.81 \\
(41.58)\end{array}$ & 63.9 & $\begin{array}{l}157.95 \\
(37.88)\end{array}$ & $58.5^{\mathrm{c}}$ & $\begin{array}{l}208.61 \\
(33.65)\end{array}$ & 76.1 & $\begin{array}{l}138.13 \\
(37.63)\end{array}$ & $51.6^{\mathrm{c}}$ \\
\hline $\begin{array}{l}\text { Light physical activ- } \\
\text { ity }\end{array}$ & $\begin{array}{l}83.13 \\
(25.71)\end{array}$ & $30.4^{\mathrm{d}}$ & $\begin{array}{l}100.84 \\
(39.53)\end{array}$ & $34.6^{\mathrm{d}}$ & $\begin{array}{l}47.47 \\
(12.97)\end{array}$ & 16.7 & $\begin{array}{l}60.11 \\
(26.45)\end{array}$ & 21.2 & $\begin{array}{l}89.25 \\
(23.68)\end{array}$ & $31.3^{\mathrm{d}}$ \\
\hline $\begin{array}{l}\text { Moderate physical } \\
\text { activity }\end{array}$ & $\begin{array}{l}19.27 \\
(10.95)\end{array}$ & 7.1 & $\mathrm{~N} / \mathrm{A}$ & N/A & $9.20(3.44)$ & 3.3 & $3.73(2.18)$ & 1.3 & $\begin{array}{l}41.46 \\
(19.87)\end{array}$ & 14.7 \\
\hline $\begin{array}{l}\text { Vigorous physical } \\
\text { activity }\end{array}$ & $\begin{array}{l}13.24 \\
(20.74)\end{array}$ & $4.7^{\mathrm{e}}$ & N/A & N/A & $\begin{array}{l}60.84 \\
(28.09)\end{array}$ & 21.5 & $3.82(2.88)$ & 1.4 & $6.74(4.42)$ & $2.4^{\mathrm{e}}$ \\
\hline $\begin{array}{l}\text { Moderate-to-vigor- } \\
\text { ous physical activity }\end{array}$ & $\begin{array}{l}32.61 \\
(30.78)\end{array}$ & 11.8 & $4.35(3.16)$ & 1.5 & $\begin{array}{l}70.98 \\
(61.63)\end{array}$ & 24.8 & $7.62(4.77)$ & 2.7 & $\begin{array}{l}48.87 \\
(23.47)\end{array}$ & 17.1 \\
\hline
\end{tabular}

${ }^{\mathrm{a}} \mathrm{ROC}$ : Receiver operating characteristic.

${ }^{\mathrm{b}}$ OLR: Ordinal logistic regression.

${ }^{\mathrm{c}}$ Indicates there is no statistical difference in PA estimate between the ML method and the hip-based reference for the Sedentary behavior intensity level.

$\mathrm{d}$ Indicates there is no statistical difference in PA estimate between the ML method and the hip-based reference for the Sedentary behavior intensity level.

e Indicates there is no statistical difference in PA estimate between the ML method and the hip-based reference for the Sedentary behavior intensity level.

\section{Discussion}

This study applied wrist derived VM data to assess PA in preschoolers based on three ML models. In the supervised ML models, the ROC analysis and OLR model were able to distinguish VM counts into each PA intensity level by reading the intensity label of each epoch as assigned by the gold standard hip reference. As an unsupervised ML model, k-means cluster analysis successfully grouped accelerometer count values into four PA clusters. When examining the agreement and comparing PA estimates from the wrist data compared with the hip reference, the k-means cluster analysis had the best performance among three ML models tested. Additionally, the k-means method of assigning PA levels had better agreement with the hip reference than the previously published cut points developed by Johansson et al [25].
Our results showed that cut points derived from the k-means cluster analysis produced better agreement with the gold standard hip reference than the other two ML approaches across all sedentary behavior and PA intensity levels, as indicated by sensitivity, specificity, and kappa values. K-means sedentary behavior, LPA, and VPA cut points showed the highest similarity to the hip reference on estimating PA time. Additionally, the estimated percent time in sedentary behavior, LPA, and MVPA by the k-means wrist reference method was comparable to the findings by Jones et al [35], who adopted a hip-worn accelerometry protocol for assessing PA in preschoolers similar to the one used in this study.

One reason that the k-means method had superior performance could be that the use of unlabeled data for calibration may result in less biased cut points for classifying epochs into PA levels [36]. During the data training process for the supervised learning 
models, the hip reference-based PA levels were used as "labels" or "targets," which the models are trying to use as a basis to classify each epoch into the "true PA state." Since most of the epochs in our calibration data were observed in the sedentary state based on the hip reference, this could result in biasing the supervised model cut points toward lower activity levels. Thus, the lower activity levels might overly influence supervised learning models when developing cut points for wrist-worn accelerometers.

Similarly, the nature of the activities generating the calibration data may also influence the performance of the machine learning methods. For example, Butte et al [24] used 11 structured activities as the ground truth activity to calibrate the cut points for the hip reference [37]. However, others have found that using free-living activities for accelerometer calibration might generate higher counts per minute cut points than structured activities [38]. This might explain the higher MVPA cut point $(\geq 16716 \mathrm{cpm})$ developed by Johansson et al [25], which incorporated free-play sessions during the calibration process, compared to our calibration data, which used only structured activities. Regardless, the k-means method showed superior performance in accurately assessing PA levels compared to the ROC, OLR and Johansson et al [25] methods. Thus, the k-means approach represents an improvement on existing methods for establishing wrist-based PA levels among pre-school aged children.

Our results showed that all wrist-based methods had difficulty in accurately assessing MPA. One possible explanation for this issue is that there is no consensus on what types of activities can represent MPA for this age group [25]. For example, the Johansson study [25] used a ball-toss activity, a 10-minute active video game, a 15-minute dancing activity, and an aerobic video activity to represent MPA, whereas Pate et al [39] defined the intensity between slow and brisk walking as the cut point for MPA and Sirard et al [40] used fast walking at 4.3 (SD 0.6) $\mathrm{km} / \mathrm{h}$ as their criterion activity to represent MPA. Therefore, combined criterion-based and free-living activities for the generation of model training data may better reflect the full range of MPA, which could result in improved calibration processes for children in this age group. Future studies would also benefit from additional analysis of raw accelerometry data from wrist-worn devices with unsupervised learning methods.

\section{Limitations}

This study has several limitations. First, participants in this study were from low-income minority families and, therefore, accelerometer counts collected from the study sample might not represent the activity patterns of the general population. Previous studies have shown that young children from low-income minority families had a significantly lower motor performance and are less active during a preschool day compared to children from higher income families [41,42]. However, previous research found no difference in cut points in older children and adults from different income and socioeconomic background [13]. Second, this study chose a 30 Hertz sampling rate for both hip and wrist accelerometer placement sites to collect raw acceleration data to make the results comparable to other studies, but this approach might lead to an underestimate of activity intensity [13]. For instance, Clevenger et al [43] found that greater sampling rate resulted in a higher estimation of high-intensity PA in preschoolers for both hip- and wrist-worn accelerometers, even though the difference was not significant. Thus, the study should be replicated using higher sampling rate magnitude. Another limitation is that this study collected data during school hours on 3 days, which may not reflect the general PA patterns of preschool-aged children. Hesketh et al [44] found that children were more active in daycare than at home and were more active during weekdays than weekends days. In this case, collecting accelerometry data from both weekdays and weekend days based on a 24-hour accelerometry protocol would reflect the PA pattern in this age group more accurately. Finally, the cut points from ML models were not validated against ground truth activities that can substantiate the accuracy of the wrist-derived data [16].

\section{Conclusion}

This study demonstrated the potential of ML techniques to distinguish PA intensity levels, with the exception of MPA, in preschool-age children. Cut point established from k-means cluster analysis was comparable to the hip-reference criterion in predicting sedentary behavior. Although PA estimates from k-means cluster analysis of wrist-worn accelerometers were acceptable as compared to the hip reference, the finding needs to be replicated using ground truth activities in free-living setting.

\section{Acknowledgments}

This study is funded by the United States National Institutes of Health, National Institute of Diabetes and Digestive and Kidney Diseases (R01DK109323). Parent/Child Incorporated Of San Antonio And Bexar and Family Service Association of San Antonio, Inc, are the study collaborators who administer Head Start Programs in San Antonio, Texas. Our heartfelt appreciation goes to the children, parents, and staff of Head Start Programs who participated in our study. Finally, we want to thank our research staff, undergraduate intern students, and graduate students for their hard work and contribution to the study.

\section{Authors' Contributions}

ZY and SL contributed to the design and implementation of the study. JH and SL conducted the statistical analyses of this work. SL took the lead in writing manuscript. All authors participated in the implementation of the study protocol, helped interpret the results, contributed to the discussion and approved the final manuscript. 


\section{Conflicts of Interest}

None declared.

\section{References}

1. Matthews CE, Hagströmer M, Pober DM, Bowles HR. Best practices for using physical activity monitors in population-based research. Med Sci Sports Exerc 2012 Jan;44(1 Suppl 1):S68-S76 [FREE Full text] [doi: 10.1249/MSS.0b013e3182399e5b] [Medline: 22157777]

2. Freedson P, Bowles HR, Troiano R, Haskell W. Assessment of physical activity using wearable monitors: recommendations for monitor calibration and use in the field. Med Sci Sports Exerc 2012 Jan;44(1 Suppl 1):S1-S4 [FREE Full text] [doi: 10.1249/MSS.0b013e3182399b7e] [Medline: 22157769]

3. Bornstein DB, Beets MW, Byun W, McIver K. Accelerometer-derived physical activity levels of preschoolers: a meta-analysis. J Sci Med Sport 2011 Nov;14(6):504-511. [doi: 10.1016/j.jsams.2011.05.007] [Medline: 21684809]

4. Chen KY, Bassett DR. The technology of accelerometry-based activity monitors: current and future. Med Sci Sports Exerc 2005 Nov;37(11 Suppl):S490-S500. [doi: 10.1249/01.mss.0000185571.49104.82] [Medline: 16294112]

5. Bochniewicz EM, Emmer G, McLeod A, Barth J, Dromerick AW, Lum P. Measuring Functional Arm Movement after Stroke Using a Single Wrist-Worn Sensor and Machine Learning. J Stroke Cerebrovasc Dis 2017 Dec;26(12):2880-2887. [doi: 10.1016/j.jstrokecerebrovasdis.2017.07.004] [Medline: 28781056]

6. Ellis K, Kerr J, Godbole S, Staudenmayer J, Lanckriet G. Hip and Wrist Accelerometer Algorithms for Free-Living Behavior Classification. Med Sci Sports Exerc 2016 May;48(5):933-940 [FREE Full text] [doi: 10.1249/MSS.0000000000000840] [Medline: 26673126]

7. Huberty J, Ehlers DK, Kurka J, Ainsworth B, Buman M. Feasibility of three wearable sensors for 24 hour monitoring in middle-aged women. BMC Womens Health 2015 Jul 30;15:55 [FREE Full text] [doi: 10.1186/s12905-015-0212-3] [Medline: 26223521]

8. Full KM, Kerr J, Grandner MA, Malhotra A, Moran K, Godoble S, et al. Validation of a physical activity accelerometer device worn on the hip and wrist against polysomnography. Sleep Health 2018 Apr;4(2):209-216 [FREE Full text] [doi: 10.1016/j.sleh.2017.12.007] [Medline: 29555136]

9. Kwon S, Zavos P, Nickele K, Sugianto A, Albert MV. Hip and Wrist-Worn Accelerometer Data Analysis for Toddler Activities. Int J Environ Res Public Health 2019 Jul 21;16(14) [FREE Full text] [doi: 10.3390/ijerph16142598] [Medline: $\underline{31330889]}$

10. Fairclough SJ, Noonan R, Rowlands AV, Van Hees V, Knowles Z, Boddy LM. Wear compliance and activity in children wearing wrist- and hip-mounted accelerometers. Med Sci Sports Exerc 2016 Feb;48(2):245-253. [doi: 10.1249/MSS.0000000000000771] [Medline: 26375253]

11. Kim Y, Beets MW, Welk GJ. Everything you wanted to know about selecting the "right" Actigraph accelerometer cut-points for youth, but...: a systematic review. J Sci Med Sport 2012 Jul;15(4):311-321. [doi: 10.1016/j.jsams.2011.12.001] [Medline: 22306372]

12. Cain KL, Sallis JF, Conway TL, Van Dyck D, Calhoon L. Using accelerometers in youth physical activity studies: a review of methods. J Phys Act Health 2013 Mar;10(3):437-450 [FREE Full text] [doi: 10.1123/jpah.10.3.437] [Medline: 23620392]

13. Migueles JH, Cadenas-Sanchez C, Ekelund U, Delisle Nyström C, Mora-Gonzalez J, Löf M, et al. Accelerometer Data Collection and Processing Criteria to Assess Physical Activity and Other Outcomes: A Systematic Review and Practical Considerations. Sports Med 2017 Sep;47(9):1821-1845 [FREE Full text] [doi: 10.1007/s40279-017-0716-0] [Medline: $\underline{28303543]}$

14. Johansson E, Ekelund U, Nero H, Marcus C, Hagströmer M. Calibration and cross-validation of a wrist-worn Actigraph in young preschoolers. Pediatr Obes 2015 Feb;10(1):1-6. [doi: 10.1111/j.2047-6310.2013.00213.x] [Medline: 24408275]

15. Hislop J, Palmer N, Anand P, Aldin T. Validity of wrist worn accelerometers and comparability between hip and wrist placement sites in estimating physical activity behaviour in preschool children. Physiol Meas 2016 Oct;37(10):1701-1714. [doi: 10.1088/0967-3334/37/10/1701] [Medline: 27653188]

16. Farrahi V, Niemelä M, Kangas M, Korpelainen R, Jämsä T. Calibration and validation of accelerometer-based activity monitors: A systematic review of machine-learning approaches. Gait Posture 2019 Feb;68:285-299 [FREE Full text] [doi: 10.1016/j.gaitpost.2018.12.003] [Medline: 30579037]

17. Koza J, Bennett III FH, Andre D, Keane MA. Automated design of both the topology and sizing of analog electrical circuits using genetic programming. In: Gero JS, Sudweeks F, editors. Artificial intelligence in design '96. Dordrecht: Kluwer Academic Publishers; 1996:151-170.

18. Ellis K, Kerr J, Godbole S, Lanckriet G, Wing D, Marshall S. A random forest classifier for the prediction of energy expenditure and type of physical activity from wrist and hip accelerometers. Physiol Meas 2014 Nov;35(11):2191-2203 [FREE Full text] [doi: 10.1088/0967-3334/35/11/2191] [Medline: 25340969]

19. Narayanan A, Desai F, Stewart T, Duncan S, Mackay L. Application of raw accelerometer data and machine-learning techniques to characterize human movement behavior: a systematic scoping review. J Phys Act Health 2020 Mar 01;17(3):360-383. [doi: 10.1123/jpah.2019-0088] [Medline: 32035416] 
20. Scott JJ, Rowlands AV, Cliff DP, Morgan PJ, Plotnikoff RC, Lubans DR. Comparability and feasibility of wrist- and hip-worn accelerometers in free-living adolescents. J Sci Med Sport 2017 Dec;20(12):1101-1106. [doi: 10.1016/j.jsams.2017.04.017] [Medline: 28501418]

21. Tudor-Locke C, Barreira TV, Schuna JM, Mire EF, Chaput J, Fogelholm M, ISCOLE Research Group. Improving wear time compliance with a 24-hour waist-worn accelerometer protocol in the International Study of Childhood Obesity, Lifestyle and the Environment (ISCOLE). Int J Behav Nutr Phys Act 2015 Feb 11;12:11 [FREE Full text] [doi: 10.1186/s12966-015-0172-x] [Medline: 25881074]

22. Hinkley T, Crawford D, Salmon J, Okely AD, Hesketh K. Preschool children and physical activity: a review of correlates. Am J Prev Med 2008 May;34(5):435-441. [doi: 10.1016/j.amepre.2008.02.001] [Medline: 18407012]

23. Rowlands A. Moving forward with accelerometer-assessed physical activity: two strategies to ensure meaningful, interpretable, and comparable measures. Pediatr Exerc Sci 2018 Nov 01;30(4):450-456. [doi: 10.1123/pes.2018-0201] [Medline: 30304982]

24. Butte NF, Wong WW, Lee JS, Adolph AL, Puyau MR, Zakeri IF. Prediction of energy expenditure and physical activity in preschoolers. Med Sci Sports Exerc 2014 Jun;46(6):1216-1226 [FREE Full text] [doi: 10.1249/MSS.0000000000000209] [Medline: 24195866]

25. Johansson E, Larisch L, Marcus C, Hagströmer M. Calibration and validation of a wrist- and hip-worn actigraph accelerometer in 4-year-old children. PLoS One 2016;11(9):e0162436 [FREE Full text] [doi: 10.1371/journal.pone.0162436] [Medline: 27617962]

26. Kingsley MI, Nawaratne R, O'Halloran PD, Montoye AH, Alahakoon D, De Silva D, et al. Wrist-specific accelerometry methods for estimating free-living physical activity. J Sci Med Sport 2019 Jun;22(6):677-683. [doi: 10.1016/j.jsams.2018.12.003] [Medline: 30558904]

27. Cliff DP, Reilly JJ, Okely AD. Methodological considerations in using accelerometers to assess habitual physical activity in children aged 0-5 years. J Sci Med Sport 2009 Sep;12(5):557-567. [doi: 10.1016/j.jsams.2008.10.008] [Medline: 19147404]

28. Choi L, Liu Z, Matthews CE, Buchowski MS. Validation of accelerometer wear and nonwear time classification algorithm. Med Sci Sports Exerc 2011 Feb;43(2):357-364 [FREE Full text] [doi: 10.1249/MSS.0b013e3181ed61a3] [Medline: 20581716]

29. Alpaydin E. Introduction to machine learning. Cambridge: The MIT Press; 2020.

30. Zou KH, O'Malley AJ, Mauri L. Receiver-operating characteristic analysis for evaluating diagnostic tests and predictive models. Circulation 2007 Feb 06;115(5):654-657. [doi: 10.1161/CIRCULATIONAHA.105.594929] [Medline: 17283280]

31. Alhassan S, Robinson TN. Defining accelerometer thresholds for physical activity in girls using ROC analysis. J Phys Act Health 2010 Jan;7(1):45-53 [FREE Full text] [doi: 10.1123/jpah.7.1.45] [Medline: 20231754]

32. Fraley C. How many clusters? Which clustering method? Answers via model-based cluster analysis. Comput J 1998 Aug 01;41(8):578-588. [doi: 10.1093/comjnl/41.8.578]

33. Jain AK. Data clustering: 50 years beyond K-means. Pattern Recognit Lett 2010 Jun;31(8):651-666. [doi: 10.1016/j.patrec.2009.09.011]

34. Wilks DS. Statistical methods in the atmospheric sciences. Cambridge: Academic Press; 2011.

35. Jones RA, Okely AD, Hinkley T, Batterham M, Burke C. Promoting gross motor skills and physical activity in childcare: A translational randomized controlled trial. J Sci Med Sport 2016 Sep;19(9):744-749. [doi: 10.1016/j.jsams.2015.10.006] [Medline: 26774378]

36. van Kuppevelt D, Heywood J, Hamer M, Sabia S, Fitzsimons E, van Hees V. Segmenting accelerometer data from daily life with unsupervised machine learning. PLoS One 2019;14(1):e0208692 [FREE Full text] [doi:

10.1371/journal.pone.0208692] [Medline: 30625153]

37. Zakeri I, Adolph AL, Puyau MR, Vohra FA, Butte NF. Cross-sectional time series and multivariate adaptive regression splines models using accelerometry and heart rate predict energy expenditure of preschoolers. J Nutr 2013 Jan;143(1):114-122 [FREE Full text] [doi: 10.3945/jn.112.168542] [Medline: 23190760]

38. van Cauwenberghe E, Labarque V, Trost SG, de Bourdeaudhuij I, Cardon G. Calibration and comparison of accelerometer cut points in preschool children. Int J Pediatr Obes 2011 Jun;6(2-2):e582-e589. [doi: 10.3109/17477166.2010.526223] [Medline: 21121867]

39. Pate RR, Almeida MJ, McIver KL, Pfeiffer KA, Dowda M. Validation and calibration of an accelerometer in preschool children. Obesity (Silver Spring) 2006 Nov;14(11):2000-2006 [FREE Full text] [doi: 10.1038/oby.2006.234] [Medline: 17135617]

40. Sirard JR, Trost SG, Pfeiffer KA, Dowda M, Pate RR. Calibration and evaluation of an objective measure of physical activity in preschool children. J Phys Act Health 2005 Jul;2(3):345-357. [doi: 10.1123/jpah.2.3.345]

41. Iivonen S, Sääkslahti A, Mehtälä A, Villberg J, Soini A, Poskiparta M. Directly observed physical activity and fundamental motor skills in four-year-old children in day care. Eur Early Child Educ Res J 2016 Apr 21;24(3):398-413. [doi: 10.1080/1350293x.2016.1164398]

42. Innella N, Breitenstein S, Hamilton R, Reed M, McNaughton DB. Determinants of obesity in the hispanic preschool population: an integrative review. Public Health Nurs 2016 May;33(3):189-199. [doi: 10.1111/phn.12215] [Medline: 26118340] 
43. Clevenger KA, Pfeiffer KA, Mackintosh KA, McNarry MA, Brønd J, Arvidsson D, et al. Effect of sampling rate on acceleration and counts of hip- and wrist-worn ActiGraph accelerometers in children. Physiol Meas 2019 Sep 30;40(9):095008. [doi: 10.1088/1361-6579/ab444b] [Medline: $\underline{31518999]}$

44. Hesketh KR, Griffin SJ, van Sluijs EMF. UK Preschool-aged children's physical activity levels in childcare and at home: a cross-sectional exploration. Int J Behav Nutr Phys Act 2015 Sep 26;12(1). [doi: 10.1186/s12966-015-0286-1]

\author{
Abbreviations \\ LPA: light physical activity \\ ML: machine learning \\ MPA: moderate physical activity \\ MVPA: moderate-to-vigorous physical activity \\ OLR: ordinal logistic regression \\ PA: physical activity \\ ROC: receiver operating characteristic \\ VPA: vigorous physical activity
}

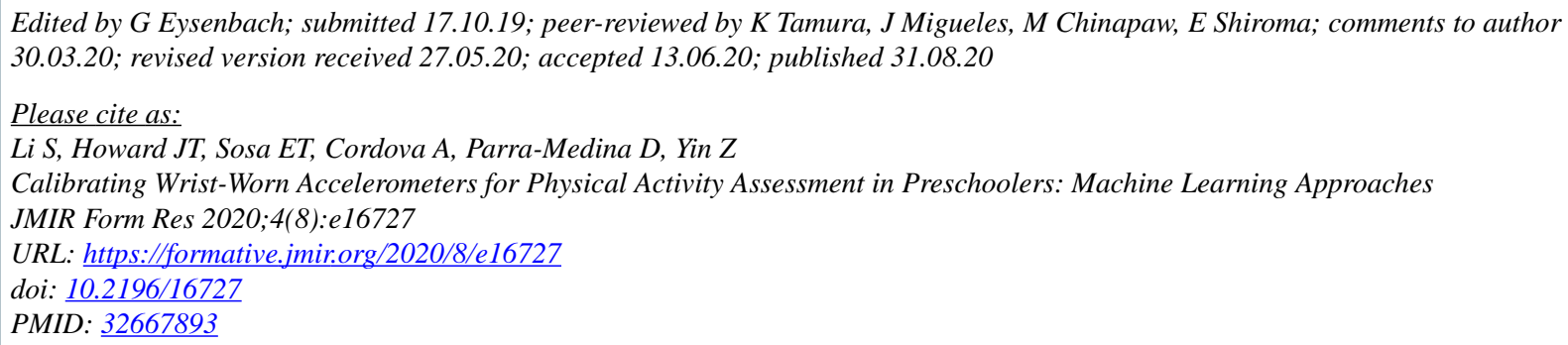

(CShiyu Li, Jeffrey T Howard, Erica T Sosa, Alberto Cordova, Deborah Parra-Medina, Zenong Yin. Originally published in JMIR Formative Research (http://formative.jmir.org), 31.08.2020. This is an open-access article distributed under the terms of the Creative Commons Attribution License (https://creativecommons.org/licenses/by/4.0/), which permits unrestricted use, distribution, and reproduction in any medium, provided the original work, first published in JMIR Formative Research, is properly cited. The complete bibliographic information, a link to the original publication on http://formative.jmir.org, as well as this copyright and license information must be included. 